\title{
Encefalomielitis aguda diseminada grave. Comunicación de un caso asociado a infección por virus parainfluenza 3
}

\author{
Felipe Olivares, Marcia Salinas, Andrés Soto, Jeannette Dabanch y Alberto Fica
}

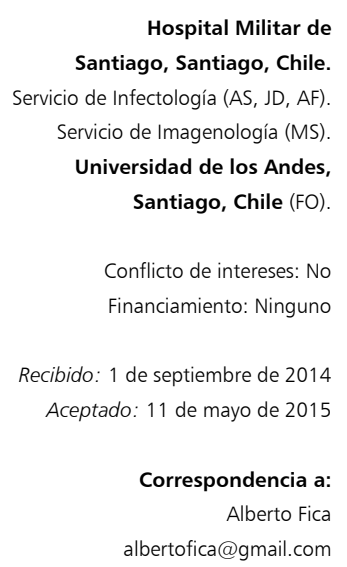

\section{Severe acute disseminated encephalomyelitis associated with parainfluenza 3 infection. Case report}

Disseminated encephalomyelitis (ADEM) is an infrequent condition with considerable morbidity and mortality in adult patients. It requires a high level of suspicion and diagnosis emerges by gathering clinical information, laboratory exams and images studies. ADEM is related to an immunological phenomena occurring after a bacterial/ viral infection or recent vaccination. Glucocorticoids are the first line treatment, reserving immunoglobulins and plasmapheresis to refractory cases. We report a male patient aged 25, with ADEM associated to parainfluenza 3 virus respiratory infection that required mechanical ventilation and that had a complete recovery only after plasmapheresis.

Key words: Acute disseminated encephalomyelitis, human parainfluenza virus 3, adult, plasmapheresis, glucocorticoids, intravenous immunoglobulins.

Palabras clave: Encefalomielitis aguda diseminada, virus parainfluenza humano 3, adulto, plasmaféresis, glucocorticoides, inmunoglobulinas endovenosas.

\section{Introducción}

L a encefalomielitis aguda diseminada (EMAD), también conocida como encefalitis post-infecciosa, es una enfermedad inflamatoria mediada inmunológicamente, de baja frecuencia, que compromete el sistema nervioso central (SNC). Afecta especialmente a niños y adolescentes, no presenta diferencias según género, y se manifiesta por alteraciones neurológicas multifocales, rápidamente progresivas y de presentación habitualmente monofásica, aunque en algunos casos puede haber recurrencias ${ }^{1-3}$. En 50 a $75 \%$ de los casos se ha relacionado con una infección viral, bacteriana y/o inmunización precedente ${ }^{4,5}$; sin embargo, sus mecanismos patogénicos no están dilucidados. Se presenta en forma más grave en adultos, con una mortalidad global estimada de $12 \%$, y en menos de la mitad de los casos habría una recuperación completa ${ }^{4,6,7}$. Su baja incidencia hace que el médico clínico esté poco interiorizado con esta entidad, retrasando la sospecha diagnóstica y su manejo.

Se presenta el caso clínico de una EMAD grave, asociado a una infección por virus parainfluenza 3 y además se realiza una actualización de los aspectos clínicos, terapéuticos y patogénicos de esta entidad.

\section{Caso clínico}

Paciente de sexo masculino, edad 25 años, previamente sano, refirió un cuadro autolimitado de cinco días de tos, odinofagia, fiebre, mialgias y calofríos. Después de dos días ya sin síntomas, reapareció la fiebre $\left(38^{\circ} \mathrm{C}\right)$ y desarrolló una diplopía en el ojo derecho acompañada de ataxia, disfagia leve, paresia en su extremidad superior derecha y disnea progresiva. Consultó en el Servicio de Urgencia, requiriendo rápidamente intubación y conexión a ventilación mecánica invasora (VMI). En la anamnesis complementaria refirió que había recibido la vacuna anti-influenza un mes previo al inicio del cuadro. No tenía antecedentes familiares relevantes, contactos con tóxicos ni con animales, enfermedades de transmisión sexual o conductas de riesgo para ellas, exposición a un ambiente rural, viajes fuera del país, ni síntomas sugerentes de mesenquimopatías. Al examen físico se constató obnubilación, nistagmus vertical, paresia del VI y VII pares craneales y hemiparesia braquial derecha. No hubo elevación en los parámetros inflamatorios y el líquido cefalorraquídeo (LCR) demostró 8 cél $/ \mathrm{mm}^{3}$, glucorraquia de $77 \mathrm{mg} / \mathrm{dL}$, proteínorraquia de $38 \mathrm{mg} / \mathrm{dL}$, test de tinta china negativa, adenosindeaminasa (ADA) 1,38 U/L y ausencia de bacterias a la tinción de Gram. No se realizó búsqueda de bandas oligoclonales ni tampoco de anticuerpos intratecales. La tomografía axial computada (TAC) de cerebro sin contraste no mostró alteraciones. La primera resonancia magnética (RM) cerebral mostró en secuencias T2, Flair y Difusión, múltiples focos hiperintensos en la zona entre el bulbo raquídeo y la médula espinal, en la región latero-bulbar derecha, en las zonas sub-corticales de ambas regiones temporo-insulares y en 


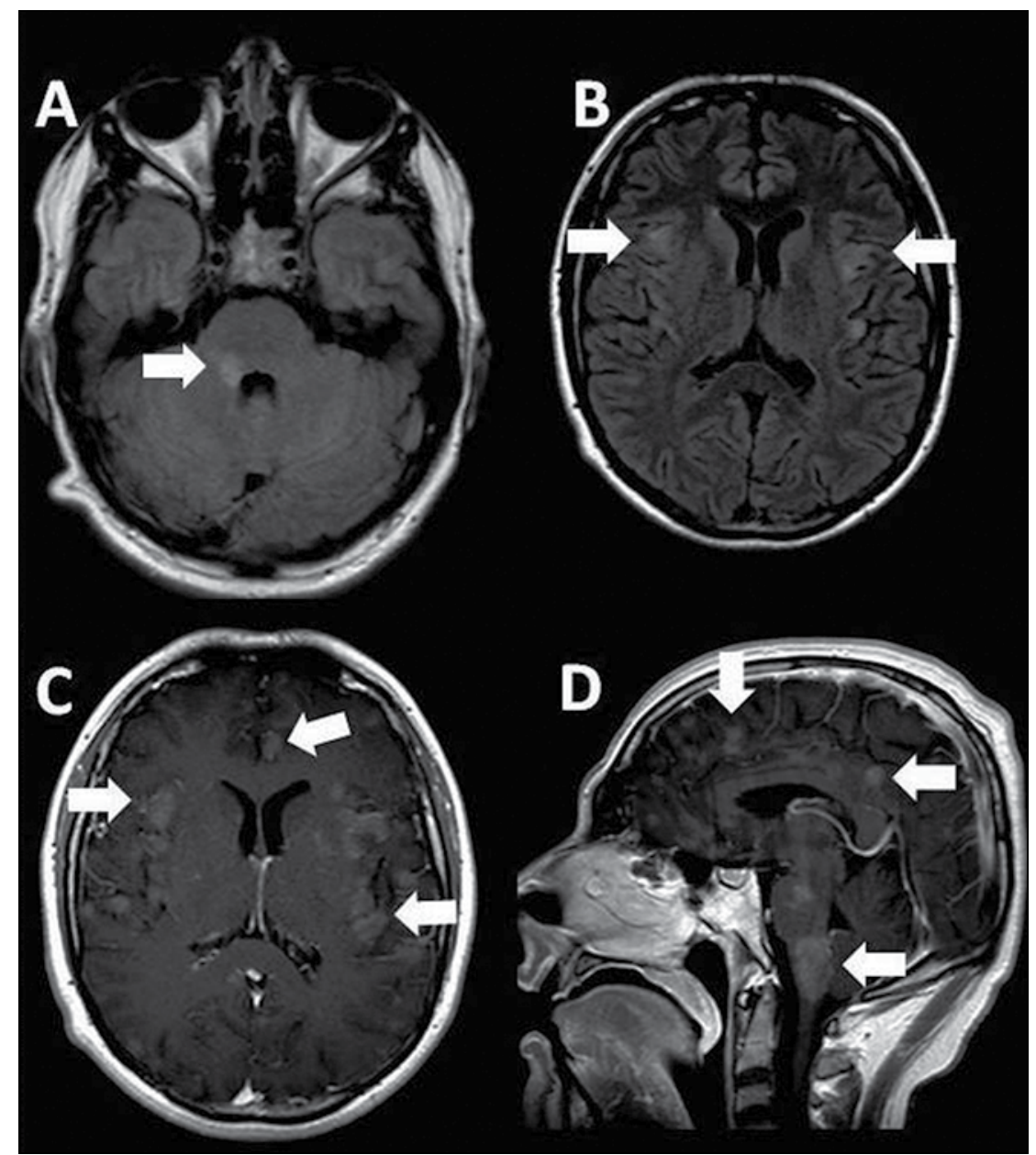

la corteza de ambas convexidades parietales (Figura 1A y 1B). Todas ellas se impregnaron con gadolinio (Gd), traduciendo actividad inflamatoria aguda (Figura 1C y 1D). Se plantearon entre los diagnósticos diferenciales: una encefalomielitis aguda diseminada, esclerosis múltiple (EM) y menos probable una vasculitis o embolías sépticas. La angioresonancia fue normal, descartando un accidente cerebrovascular agudo.

Se inició tratamiento empírico con ceftriaxona iv $2 \mathrm{~g}$ cada $12 \mathrm{~h}$, ampicilina iv $3 \mathrm{~g}$ cada $6 \mathrm{~h}$, aciclovir iv $10 \mathrm{mg} /$ $\mathrm{kg} /$ dosis cada $8 \mathrm{~h}$ y dexametasona iv. Durante las primeras $72 \mathrm{~h}$ se realizó un amplio estudio etiológico complementario: las serologías para sífilis, VIH, virus de Epstein Barr (VEB), citomegalovirus (CMV), Mycoplasma pneumoniae, Brucella spp. y Chlamydia spp. en muestras de sangre fueron negativas. Además los hemocultivos, el examen toxicológico para diferentes compuestos (cocaína, canabinoides, anfetamina y metanfetamina), los anticuerpos anti-nucleares y anti-citoplasma de neutrófilos también resultaron negativos. Las pruebas tiroideas y el recuento de inmunoglobulinas estaban en rangos normales y no hubo alteraciones en la TAC de tórax-abdomen y pelvis, ni tampoco se evidenciaron vegetaciones en el ecocardiograma transesofágico. Paralelamente, el estudio específico del LCR no mostró crecimiento bacteriano y resultó negativo para virus herpes simplex 1 y 2 (VHS 1 y 2), varicela zoster (VVZ), CMV y VEB por RPC. Se detectó virus parainfluenza 3 por inmunofluorescencia mediante muestra nasofaríngea. En contraste, no hubo evidencia de otros virus respiratorios analizados (influenza A y B, virus respiratorio sincicial, parainfluenza 1 y 2 , adenovirus y metapneumovirus humano).

Tras haber excluido una meningoencefalitis viral y bacteriana se optó por suspender la terapia antibacteriana y antiviral a las $72 \mathrm{~h}$ de su inicio. En ese escenario, considerando el progresivo deterioro neurológico y los hallazgos en la RM cerebral se inició inmunoglobulina intravenosa $(0,5 \mathrm{~g} / \mathrm{kg}$ /día por cinco días), sin demostrar mejoría tras $48 \mathrm{~h}$. La RM de control a los seis días demostró progresión en número y extensión de focos hiperintensos observados en el primer examen, comprometiendo además la sustancia blanca sub-insular bilateral y ambas cápsulas internas, con caracteres desmielinizantes y compromiso de núcleos grises basales anteriores bilaterales, apoyando el diagnóstico de una EMAD. En este estudio ya no se observó impregnación de Gd (Figuras 2A, 2B y 2C). Ante la posibilidad de una EMAD se agregaron bolos intravenosos de metilprednisolona $500 \mathrm{mg}$ diarios, por tres dosis, sin respuesta clínica. Por ello, y luego de una semana de hospitalización, recibió plasmaféresis por siete sesiones, con recuperación progresiva del nivel de conciencia y déficit neurológico motor desde la primera sesión. Logró ser desconectado de la VMI y se observó una regresión imagenológica
Figura 1. RM tomada al ingreso. A. Flair en corte transverso. Se observa foco hiperintenso en hemiprotuberancia derecha (flecha). B. Flair en corte transverso. Se aprecian focos hiperintensos en ambas regiones temporo-insulares (flechas). C. Señal T1 en corte transverso con gadolinio. Se observa impregnación focal múltiple en ambas regiones temporo-insulares, corteza frontal y parietal (flechas). D. Señal T1 en corte sagital con gadolinio. Se aprecia impregnación focal múltiple destacando el compromiso del tronco cerebral y a nivel supratentorial (flechas).

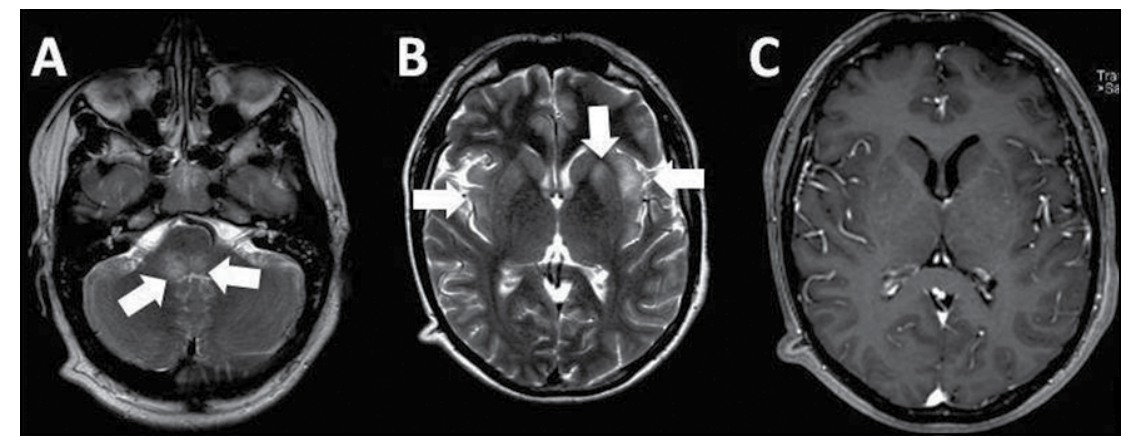

Figura 2. RM a los 6 días de hospitalización. A. Señal T2 en corte transverso. Focos hiperintensos bulbares bilaterales (flechas). B. Señal T2 en corte transverso. Focos hiperintensos en regiones temporoinsulares, sustancia blanca temporal subcortical bilateral y núcleos grises basales (flechas). C. Señal T1 en corte transverso con gadolinio.Ausencia de impregnación en todos los cortes. 


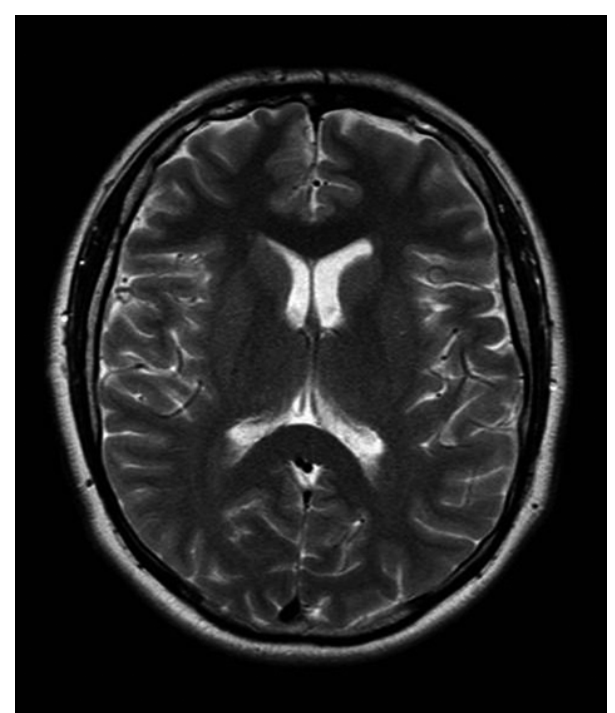

Figura 3. RM de control tardio. Señal T2 en corte transverso que revela normalización de las imágenes.

completa (Figura 3). Se dio de alta y el seguimiento a los tres años no evidenció secuelas clínicas, imagenológicas ni recurrencia.

\section{Discusión}

El caso presentado corresponde a un evento de EMAD, apoyado por elementos clínicos, de laboratorio e imágenes. Al igual que este paciente, aproximadamente la mitad de los episodios de EMAD están precedidos de una infección viral, bacteriana o inmunización previa al inicio de las manifestaciones neurológicas, siendo las infecciones respiratorias altas las más reportadas ${ }^{8}$. Los síntomas neurológicos aparecen en forma abrupta, siendo frecuente en adultos el compromiso de conciencia, déficit motor y/o sensitivo, ataxia, afasia y alteración de los nervios craneales ${ }^{9}$. La falla respiratoria es más común en adultos ${ }^{6,9} \mathrm{y}$ en nuestro caso probablemente explicada por el compromiso bulbar. Todos estos elementos clínicos fueron observados en nuestro caso, incluyendo la infección viral por parainfluenza 3 .

Tal como se advierte en este caso, el diagnóstico diferencial con una encefalitis infecciosa es complejo, especialmente cuando existe fiebre. Aunque ésta es generalmente infrecuente en adultos (menos de $15 \%)^{8}$, se ha asociado hasta en $75 \%$ de los casos graves de $\mathrm{EMAD}^{10}$. Por lo anterior, el estudio del LCR precoz es fundamental y debe incluir RPC para VHS 1 y 2, VVZ, ADA, baciloscopias y cultivo de Koch. Además, según la sospecha, puede realizarse búsqueda de VHH 6, VHH 7, CMV, VEB, CMV y enterovirus. Los hallazgos del
LCR son variables e incluso en un cuarto de los casos es normal. Aunque inespecíficos, la ausencia de agente infeccioso y la pleocitosis leve observada en este caso, son característicos y apoyan el diagnóstico de una $\mathrm{EMAD}^{6,8,9}$.

La RM es el método de elección para confirmar el diagnóstico, no existiendo criterios validados en adultos. Clásicamente se describen lesiones multifocales o extensas comprometiendo la sustancia blanca, que aparecen de gran tamaño e hiperintensas en secuencia FLAIR o potenciadas en $\mathrm{T} 2$ y poco visibles en secuencias $\mathrm{T} 1$ no contrastadas. Además puede comprometer la sustancia gris, especialmente los tálamos y ganglios basales ${ }^{11}$. Un estudio que comparó hallazgos de niños y adultos, describió en estos últimos mayor compromiso de las regiones periventriculares, y menos del cerebelo y la médula espinal. El número y extensión de las lesiones también parece ser superior en adultos ${ }^{6}$. En nuestro caso observamos la mayoría de alteraciones descritas previamente. Así, desde el inicio se detectaron focos inflamatorios de sustancia blanca y también en la sustancia gris; este último hallazgo no permitió plantear una EMAD como única posibilidad diagnóstica. La gran actividad inflamatoria fue seguida por un daño morfológico desmielinizante progresivo evidente en la RM de control. En esta última, tomada después de iniciar el tratamiento con glucocorticoides e inmunoglobulina, ya no se observó actividad inflamatoria tal como lo demuestra la ausencia de captación de Gd. Esta evolución de imágenes es concordante con el diagnóstico de EMAD. Posteriormente, la mejoría clínica, está acompañada también por la remielinización de estructuras dañadas con normalización de la imagen.

El diagnóstico diferencial debe considerar enfermedades desmielinizantes, tales como la EM, la cual en muchos casos es prácticamente indistinguible de la EMAD en su etapa inicial ${ }^{12}$ y algunos plantean que son entidades relacionadas ${ }^{13,14}$. No obstante, la evolución monofásica, la recuperación neurológica, la ausencia de lesiones residuales en los estudios de imágenes y de recurrencia, respaldan el diagnóstico de EMAD como una entidad separada, tal como fue observado en nuestro paciente. Finalmente, enfermedades como vasculitis, sarcoidosis, linfoma primario y leucoencefalopatía asociada a VIH deben investigarse según el contexto.

\section{Aspectos patogénicos}

En la patogenia de esta enfermedad, se ha involucrado la destrucción de la mielina por respuesta inmune cruzada por mimetismo molecular, gatillada por antígenos virales, bacterianos o de las vacunas ${ }^{15,16}$. En nuestro caso, aparecen dos potenciales factores involucrados: la infección precedente por parainfluenza 3 y la vacuna contra la influenza.

$\mathrm{Al}$ respecto, el compromiso del SNC asociado al virus parainfluenza 3 se expresa especialmente como eventos de meningitis aséptica en niños ${ }^{17-19}$ y rara vez se ha reportado 
una relación entre parainfluenza 3 y EMAD en otras latitudes ${ }^{20,21}$. La relación temporal entre infección viral y EMAD parece ser variable. Por ejemplo, en un caso de EMAD asociado a parainfluenza 3 se describió fiebre, cefalea y vómitos cuatro días antes de la aparición de la focalidad neurológica ${ }^{21}$. En cambio, en otro paciente adulto el cuadro neurológico se presentó seis semanas posteriores a la infección viral ${ }^{20}$. Debido a que la infección viral o bacteriana precede a la desmielinización, la probabilidad de identificar al agente etiológico está condicionada por el intervalo de tiempo entre la infección y los síntomas neurológicos. Además, la síntesis de anticuerpos intratecales es infrecuente, reduciendo aún más la probabilidad de identificación etiológica ${ }^{22}$.

Vacuna anti-influenza y EMAD. Al menos 5\% de los episodios de EMAD son atribuidos a inmunizaciones, entre ellas rabia, difteria, tétanos, polio, rubéola, sarampión, parotiditis, $B$. pertussis, hepatitis $\mathrm{B}$ e influenza ${ }^{23}$, siendo esta última la más frecuentemente vinculada. En una revisión de Pellegrino y cols. ${ }^{24}$, la vacuna antiinfluenza representó $32 \%$ de todas las EMAD asociados a inmunizaciones en mayores de 18 años. No obstante, los mismos autores reconocen que no es posible establecer claramente una causalidad, considerando las limitaciones que tiene este tipo de vigilancia de eventos adversos potencialmente asociados a vacunas ${ }^{16,25}$. Para estos fines, un comité de expertos de la Organización Mundial de la Salud (OMS) ha propuesto algunos criterios que categorizan al evento adverso en alguna de las siguientes categorías graduales: "muy posible o certero", "probable", "posible", "improbable", "no relacionado" y "no clasificable". Estos criterios de aceptación internacional se basan principalmente en la frecuencia de ocurrencia del evento adverso, plausibilidad biológica, intervalo de tiempo compatible y existencia de otra condición que pudiese explicar el evento adverso ${ }^{27}$. Aplicando estos criterios a estudios de este tipo, se estima que menos de $50 \%$ de los casos atribuidos a inmunización anti-influenza pueden apenas ser catalogados como "posibles" lo que debilita una relación causal $^{28,29}$. La incidencia de complicaciones neurológicas graves post vacuna anti-influenza, incluyendo la EMAD, es muy baja y ha sido estimada en menos de $0,01 \%$ en un estudio prospectivo de 49.138 inmunizaciones en una población militar en Francia, lo que nuevamente debilita su rol causal ${ }^{30}$. Por otra parte, un estudio experimental reciente no mostró diferencias significativas en los títulos de anticuerpos antimielina en el grupo vacunado contra influenza al compararlo con controles ${ }^{31}$, lo que disminuye su plausibilidad biológica. Algunos han considerado que debe existir una relación temporal clara entre la inmunización y la aparición del cuadro de EMAD y que la limita a un plazo de una a tres semanas (máximo un mes). Ello porque intervalos superiores abren la posibilidad de otra causa encubierta o subclínica que magnifica el riesgo de la vacuna. El estudio citado previamente de Pellegrino y cols., que reportó que más de $30 \%$ de los eventos de EMAD asociados a inmunizaciones son atribuibles a la vacuna influenza ${ }^{24}$, no utilizó los exigentes criterios propuestos por la OMS y señala que cerca de $40 \%$ de los casos ocurrieron después del primer mes, cuestionando al menos que la vacuna contra la influenza sea tan relevante en la patogenia de la EMAD.

En definitiva, considerando la baja frecuencia de EMAD post-vacunación, la débil plausibilidad biológica, el intervalo límite entre inmunización-evento, y la presencia de infección por virus parainfluenza 3 como una alternativa etiológica, se puede catalogar este evento post-inmunización como "improbable", de acuerdo a la clasificación mencionada.

\section{Aspectos terapéuticos}

A la fecha, no se ha establecido algún tratamiento estándar para pacientes adultos y la información proviene mayoritariamente de series de casos y estudios retrospectivos. Se acepta como tratamiento de primera línea el uso de glucocorticoides en dosis de 0,5-1 g de metilprednisolona al día ${ }^{1}$, con una tasa de fracaso entre 30 y $50 \%$, la cual se ha asociado a edad avanzada, mayor compromiso clínico o radiológico, presencia de mielitis, compromiso periférico, y retraso del tratamiento ${ }^{9,32}$. En nuestro medio existe limitada experiencia en adultos ${ }^{32,33}$. Vásquez y cols., reportaron hace algunos años un caso de EMAD, cuyo diagnóstico requirió la realización de biopsia estereotáctica, observando excelente respuesta a glucocorticoides ${ }^{33}$. La mitad de los casos refractarios se beneficia de inmunoglobulina intravenosa ${ }^{34,35}$. Finalmente, aunque con datos limitados en adultos, la plasmaféresis también ha sido recomendada como alternativa ante fracaso a los glucocorticoides ${ }^{36,37}$. En nuestro caso, se siguió este camino terapéutico, demostrando beneficio clínico sólo ante el uso de la plasmaféresis. Fármacos inmunosupresores como ciclosporina, metotrexato y azatriopina también han sido usados en casos refracta$\operatorname{rios}^{12,38}$. Los cuadros de EMAD en adultos tienen una elevada mortalidad global, la que ha sido estimada en $12 \%$ en general y en $25 \%$ en pacientes que ingresan a unidades críticas $^{4,10}$. Del resto que sobrevive, hasta $35 \%$ evoluciona con secuelas funcionales durante los meses de seguimiento ${ }^{10}$.

\section{Resumen}

La encefalomielitis aguda diseminada es una enfermedad infrecuente pero de elevada morbi-mortalidad en pacientes adultos. Demanda una sospecha y diagnóstico precoz que requiere el concurso de información clínica, pruebas de laboratorio y estudio de imágenes. 
De sustrato inmunológico, se puede relacionar a una infección viral, bacteriana o inmunización reciente. Los glucocorticoides son el tratamiento de elección, mientras que la inmunoglobulina intravenosa y la plasmaféresis se reservan para casos refractarios. Se presenta el caso de una encefalomielitis aguda diseminada grave, en un paciente de sexo masculino de 25 años, asociado a una infección respiratoria por virus parainfluenza 3. Requirió conexión a ventilación mecánica y tuvo una respuesta completa con plasmaféresis.

\section{Referencias bibliogáficas}

1.- Tunkel A R, Glaser C A, Bloch K C, Sejvar J J, Marra C M, Roos K L, et al. The management of encephalitis: clinical practice guidelines by the Infectious Diseases Society of America. Clin Infect Dis 2008; 47: 303-27.

2.- Leake J A, Albani S, Kao A S, Senac M O, Billman G F, Nespeca M P, et al. Acute disseminated encephalomyelitis in childhood: epidemiologic, clinical and laboratory features. Pediatr Infect Dis J 2004; 23: 756-64.

3.- Brinar V V, Poser C M. Disseminated encephalomyelitis in adults. Clin Neurol Neurosurg 2008; 110: 913-8

4.- Ketelslegers I A, Visser I E, Neuteboom R F, Boon M, Catsman-Berrevoets C E, Hintzen R Q. Disease course and outcome of acute disseminated encephalomyelitis is more severe in adults than in children. Mult Scler 2011; 17: 441-8.

5.- Noorbakhsh F, Johnson R T, Emery D, Power C. Acute disseminated encephalomyelitis: clinical and pathogenesis features. Neurol Clin 2008; 26: 759-80.

6.- $\quad$ Lin C H, Jeng J S, Hsieh S T, Yip P K, Wu R $\mathrm{M}$. Acute disseminated encephalomyelitis: a follow-up study in Taiwan. J Neurol Neurosurg Psychiatry 2007; 78: 162-7.

7.- Höllinger P, Sturzenegger M, Mathis J, Schroth G, Hess C W. Acute disseminated encephalomyelitis in adults: a reappraisal of clinical, CSF, EEG, and MRI findings. J Neurol 2002; 249: 320-9.

8.- Schwarz S, Mohr A, Knauth M, Wildemann B, Storch-Hagenlocher B. Acute disseminated encephalomyelitis: a follow-up study of 40 adult patients. Neurology 2001; 56: 1313-8.

9.- Sundar U, Shrivastava M S. Acute disseminated encephalomyelitis, a prospective study of clinical profile and in-hospital outcome predictors. J Assoc Physicians India 2012; 60: 21-6.

10.- Sonneville R, Demeret S, Klein I, Bouadma L, Mourvillier B, Audibert J, et al Acute disseminated encephalomyelitis in the intensive care unit: clinical features and outcome of 20 adults. Intensive Care Med 2008; 34: 528-32.

11.- Alper G. Acute disseminated encephalomyelitis. J Child Neurol 2012; 27: 1408-25.

12.- De Seze J, Debouverie M, Zephir H, Lebrun
C, Blanc F, Bourg V, et al. Acute fulminant demyelinating disease: a descriptive study of 60 patients. Arch Neurol 2007; 64: 1426-32.

13.- Young N P, Weinshenker B G, Lucchinetti C F. Acute disseminated encephalomyelitis: current understanding and controversies. Semin Neurol 2008; 28: 84-94.

14.- Dale R C, Branson J A. Acute disseminated encephalomyelitis or multiple sclerosis: can the initial presentation help in establishing a correct diagnosis? Arch Dis Child 2005; 90: 636-9.

15.- Pietiläinen-Nicklén J, Virtanen $O$, Kolehmainen P, Uotila L, Salonen O, Lappalainen $\mathrm{M}$, et al. ADEM and virus infection. J Clin Virol 2012; 53: 2-5.

16.- Sonneville R, Klein I, de Broucker T, Wolff M. Post-infectious encephalitis in adults: diagnosis and management. J Infect 2009; 58 : 321-8.

17.- McCarthy V P, Zimmerman A W, Miller C A. Central nervous system manifestations of parainfluenza virus type 3 infections in childhood. Pediatr Neurol 1990; 6: 197-201.

18.- Arguedas A, Stutman H R, Blanding J G. Parainfluenza type 3 meningitis: report of two cases and review of the literature. Clin Pediatr (Phila) 1990; 29: 175-8.

19.- Arisoy E S, Demmler G J, Thakar S, Doerr C. Meningitis due to parainfluenza virus type 3: report of two cases and review. Clin Infect Dis 1993; 17: 995-7.

20.- Au W Y, Lie A K, Cheung R T, Cheng P W, Ooi C G, Yujenc K Y, et al. Acute disseminated encephalomyelitis after parainfluenza infection post bone marrow transplantation. Leuk Lymphoma 2002; 43: 455-7.

21. Voudris K A, Vagiakou E A, Skardoutsou A. Acute disseminated encephalomyelitis associated with parainfluenza virus infection of childhood. Brain Dev 2002; 24: 112-4.

22.- Johnson R T. The pathogenesis of acute viral encephalitis and postinfectious encephalomyelitis. J Infect Dis 1987; 155: 359-64.

23.- Huynh W, Cordato D J, Kehdi E, Masters L T, Dedousis C. Post-vaccination encephalomyelitis: literature review and illustrative case. J Clin Neurosci 2008; 15 : 1315-22.

24.- Pellegrino P, Carnovale C, Perrone V, Pozzi M, Antoniazzi S, Clementi E, et al. Acute disseminated encephalomyelitis onset: evaluation based on vaccine adverse events reporting systems. PLoS One 2013; 8: e77766.

25.- Vaccine Adverse Event Reporting System (VAERS). Disponible en: https://vaers.hhs.gov/ index. [Consultado: 21 de julio de 2014].

26.- European database of suspected adverse drug reaction reports. Disponible en: http://www. adrreports.eu. [Consultado: 21 de julio de 2014].

27.- Collet J P, MacDonald N, Cashman N, Pless R. Monitoring signals for vaccine safety: the assessment of individual adverse event reports by an expert advisory committee. Advisory Committee on Causality Assessment. Bull World Health Organ 2000; 78: 178-85.

28.- Williams S E, Pahud B A, Vellozzi C, Donofrio P D, Dekker C L, Halsey N, et al. Causality assessment of serious neurologic adverse events following $2009 \mathrm{H} 1 \mathrm{~N} 1$ vaccination. Vaccine 2011; 29: 8302-8.

29.- Loughlin A M, Marchant C D, Adams W, Barnett E, Baxter R, Black S, et al. Causality assessment of adverse events reported to the Vaccine Adverse Event Reporting System (VAERS). Vaccine 2012; 30: 7253-9.

30.- Mayet A, Ligier C, Gache K, Manet G, Nivoix P, Dia A, et al. Adverse events following pandemic influenza vaccine Pandemrix ${ }^{\circledR}$ reported in the French military forces-2009-2010. Vaccine 2011; 29: 2576-81.

31.- Stojkovic A, Kosanovic D, Maslovaric I, Jovanova-Nesic K. Role of inactivated influenza vaccine in regulation of autoimmune processes in experimental autoimmune encephalomyelitis. Int J Neurosci 2014; 124: 139-47.

32.- Guzmán J, Salinas R. Encefalomielitis aguda diseminada y colitis ulcerosa: descripción de un caso clínico. Rev Chil Neuro-Psiquiat 2012; 50 : 57-61.

33.- Vásquez P, Reccius A, Díaz M, Sáez L, Orellana P, Valdés C, et al. Encefalomielitis aguda diseminada de presentación atípica: utilidad de la biopsia estereotáctica para su diagnóstico. Caso clínico. Rev Med Chile 2006; 134: 883-6.

34.- Marchioni E, Ravaglia S, Montomoli C, Tavazzi E, Minoli L, Baldanti F, et al Postinfectious neurologic syndromes: a prospective cohort study. Neurology 2013; 80: 882-9.

35.- Ravaglia S, Piccolo G, Ceroni M, Franciotta D, Pichiecchio A, Bastianello S, et al. Severe steroid-resistant post-infectious encephalomyelitis: general features and effects 
of IVIg. J Neurol 2007; 254: 1518-23.

36.- Cortese I, Chaudhry V, So Y T, Cantor F, Cornblath D R, Rae-Grant A. Evidence-based guideline update: Plasmapheresis in neurologic disorders: report of the Therapeutics and Technology Assessment Subcommittee of the
American Academy of Neurology. Neurology 2011; 76: 294-300.

37.- Keegan M, Pineda A A, McClelland R L, Darby C H, Rodríguez M, Weinshenker B G. Plasma exchange for severe attacks of CNS demyelination: predictors of response.
Neurology 2002; 58: 143-6.

38.- Taghdiri M M, Amanati A, Abdolkarimi B. Cyclosporine in the treatment of a case of fulminant and refractory acute disseminated encephalomyelitis. Iran J Pediatr 2011; 21 : 535-8. 